\title{
Lung imaging
}

\author{
Sebastian Ley ${ }^{1,2}$
}

Affiliations: ${ }^{1}$ Dept of Diagnostic and Interventional Radiology, Chirurgische Klinik Dr. Rinecker, Munich, Germany. ${ }^{2}$ Dept of Clinical Radiology, Ludwig Maximilians University, Munich, Germany.

Correspondence: Sebastian Ley, Dept of Diagnostic and Interventional Radiology, Chirurgische Klinik Dr. Rinecker, Am Isarkanal 30, 81379 Munich, Germany. E-mail: leylagmx.net

ABSTRACT Imaging of the lung is a mainstay of respiratory medicine. It provides local information about morphology and function of the lung parenchyma that is unchallenged by other noninvasive techniques. During the 2014 European Respiratory Society International Congress in Munich, Germany, a Clinical Year in Review session was held focusing on the latest developments in pulmonary imaging. This review summarises some of the main findings of peer-reviewed articles that were published in the 12-month period prior to the 2014 International Congress.

0 @ERSpublications

Imaging provides noninvasive access to thoracic morphology and function; this review summarises recent highlights http://ow.ly/KbVtd

\section{Introduction}

Imaging plays a major role in assessment of pulmonary diseases. A broad spectrum of imaging techniques is available. The main workhorse is still computed tomography (CT), which allows fast and high-resolution assessment of the lung parenchyma and surrounding structures. New insights into structural and functional interactions are found, making an in-depth analysis of the datasets clinically valuable. However, the soft tissue contrast and gain of direct functional information is still limited. For these indications magnetic resonance imaging (MRI) and nuclear medicine techniques, especially positron emission tomography (PET), are gaining more and more importance.

This review summarises the most important scientific achievements from the year prior to the 2014 European Respiratory Society International Congress. The focus of the selected articles was based on applications in daily clinical practice; therefore, interesting publications research wise, for example regarding hyperpolarised imaging, were not included in this review.

\section{Functional lung imaging}

Endobronchial treatment of emphysema is gaining importance as alternative to lung transplantation. Several techniques are available; endobronchial valves are the most frequently used technique. Alternative options include coils, glues and other agents. Greater radiographic evidence of fissure completeness has been shown to be associated with an enhanced response to treatment [1]. The authors of this study implied that incomplete or defective fissures probably represent collateral ventilation pathways between the pulmonary lobes (parenchymal bridges). Therefore, characterisation of the fissures is important for optimal target determination in endobronchial valve treatment. Many studies using different techniques and methodologies have already demonstrated that the pulmonary fissures are highly variable among individuals, and that the interlobar fissures are frequently incomplete. However, these studies differ greatly with regard to the severity (percentage) of fissural defects, the prevalence of incomplete and accessory pulmonary fissures and their relationship with pulmonary diseases. The severity of fissural defects has been showed to vary from short gaps of few centimetres to almost complete absence [2]. One recent study addressing this issue used high

Received: Nov 192014 | Accepted after revision: Dec 222014

Conflict of interest: None declared.

Provenance: Submitted article, peer reviewed.

Copyright OERS 2015. ERR articles are open access and distributed under the terms of the Creative Commons Attribution Non-Commercial Licence 4.0. 
spatial resolution CT with multidetector CT acquisitions and overlapping thin slices in the z-axis (1.25 mm) [2]. Only $4 \%$ of fissures were found to be complete. In this study, which included patients from all Global Initiative for Chronic Obstructive Lung Disease (GOLD) stages, the prevalence and severity of fissural defects were not statistically different between the GOLD functional categories of chronic obstructive pulmonary disease (COPD). Evaluation found 81, 89 and 50\% of right oblique, right horizontal and left oblique fissures to be incomplete. A fissural defect of $<10 \%$ was present in $30 \%$ of patients. Accessory fissures were found in $16 \%$ of patients, with the left horizontal being the most common location [2].

These findings were supported by another study looking at 573 CT examinations in COPD patients [3]. While the above-mentioned study relied on a visual assessment, Pu et al. [3] used an automatic tool for assessment of fissure integrity. The integrities of the right major fissure and the left major fissure were similar $(\sim 82 \%)$, irrespective of COPD severity, and the integrity of the right minor fissure was relatively low $(\sim 62 \%)$. It can be seen that there was no obvious difference in integrity for the defined subgroups, albeit the fissure integrity for the GOLD stage IV subgroup was relatively slightly small. The fissure integrity at the entire lung level was $\sim 78 \%$ regardless of the COPD severity. In other words, $90 \%$ of the subjects had incomplete fissures. Regarding the software performance, it is noted that $\sim 20 \%$ of examinations needed manual corrections and most of these were classified as moderate and severe COPD. The level of fissure integrity was compared with lung functional parameters as an indirect marker of collateral ventilation. It was found that the pulmonary fissures integrity did not affect pulmonary function and disease severity. Pu et al. [3] concluded that the development of COPD does not affect the fissure integrity and that fissure integrity alone may not contribute to the collateral ventilation.

Another area of intense investigation is the airways, especially in COPD patients. Comparison of airway wall thickness and lumen diameter between healthy controls and COPD patients is usually hampered by the fact that a comparison of airway characteristics on a spatially matched airway generation was not performed. Thus, there are studies showing reduced airway lumen dimensions and fewer peripheral airways in COPD while other studies have demonstrated the converse, for example thicker airways in COPD. In a study based on the Multi-Ethnic Study of Atherosclerosis (MESA) and the Subpopulations and Intermediate Outcomes in COPD Study (SPIROMICS) 955 COPD patients were investigated [4]. The airway data were compared with 604 control datasets. Using CT data, airway dimensions were measured for all central airway segments (generations 0-6). Case-control airway comparisons were spatially matched by generation and adjusted for demographics, body size, smoking, CT dose, per cent emphysema, airway length and lung volume. In the controls, airway wall areas at generations $1-6$ were smaller (all $\mathrm{p}<0.001$ ) when compared with COPD patients and this reduction was monotonic with increasing COPD severity $(\mathrm{p}<0.001)$ [4].

The possibility of assessing smoking induced inflammation by CT was investigated in a study involving 120 subjects [5]. 40 never-smokers, 40 smokers and 40 COPD patients were included, and CT density measurements were correlated with markers of systemic inflammation and bronchoalveolar lavage (BAL). The mean lung attenuation was higher for smokers (mean \pm SD $-857 \pm 28 \mathrm{HU}$ ) than for never-smokers $(-874 \pm 20 \mathrm{HU})$ and patients with COPD $(-876 \pm 17 \mathrm{HU})(\mathrm{p}<0.001$ for both) (fig. 1). This was also reflected when lung density was quantified as mean \pm SD percentage high density spectrum (\%HDS) (defined as $-750--900 \mathrm{HU}$ ): $44.0 \pm 5.8 \%$ in smokers, $38.3 \pm 5.8 \%$ in never-smokers and $39.1 \pm 5.8 \%$ in COPD patients ( $\mathrm{p}<0.001$ for both comparisons). There was a significant positive correlation between lung density (\%HDS) and haptoglobin in serum in both smokers and COPD patients, while IgG had a negative correlation in smokers and COPD. There was a positive correlation between lung density (\%HDS) and total cell concentration in BAL both for smokers with normal lung function $(r=0.38 ; p=0.03)$ and when smoking COPD patients were included $(\mathrm{r}=0.50 ; \mathrm{p}<0.001)$. There was no correlation between cell concentration in BAL and lung density in never-smokers. The authors conclude that smoking causes inflammation in the lungs which can be quantified noninvasively using CT density [5].

Besides assessment of morphological changes in the lung parenchyma, CT can be used to gain functional information, namely perfusion, with the inherent high spatial resolution. With the introduction of $>128$ slice CT scanners volume perfusion of a total organ became feasible (up to $21 \mathrm{~cm}$ in the z-axis with a temporal resolution of $2 \mathrm{~s}$ ). This technique (using a 128 slice CT scanner) was used to investigate lung perfusion in 32 patients (10 with emphysema, five with pulmonary embolism and 17 subjects in the control group) [6]. 15 continuous three-dimensional datasets of the lung were acquired with a temporal resolution of $2 \mathrm{~s}$ per three-dimensional dataset during first pass perfusion of the lung parenchyma. The proposed dynamic examination protocol applied on average $6 \mathrm{mSv}$ of radiation dose to the patient, while a routine chest CT (in this setting) applied $4.1 \mathrm{mSv}$. However, it has to be mentioned that the patients included suffered from oesophageal cancer and needed a standard dose staging CT.

Failure of perfusion was observed in affected segments, and markedly or slightly increased perfusion was observed in areas that were not affected by clots or emphysema. Dynamic images were analysed 


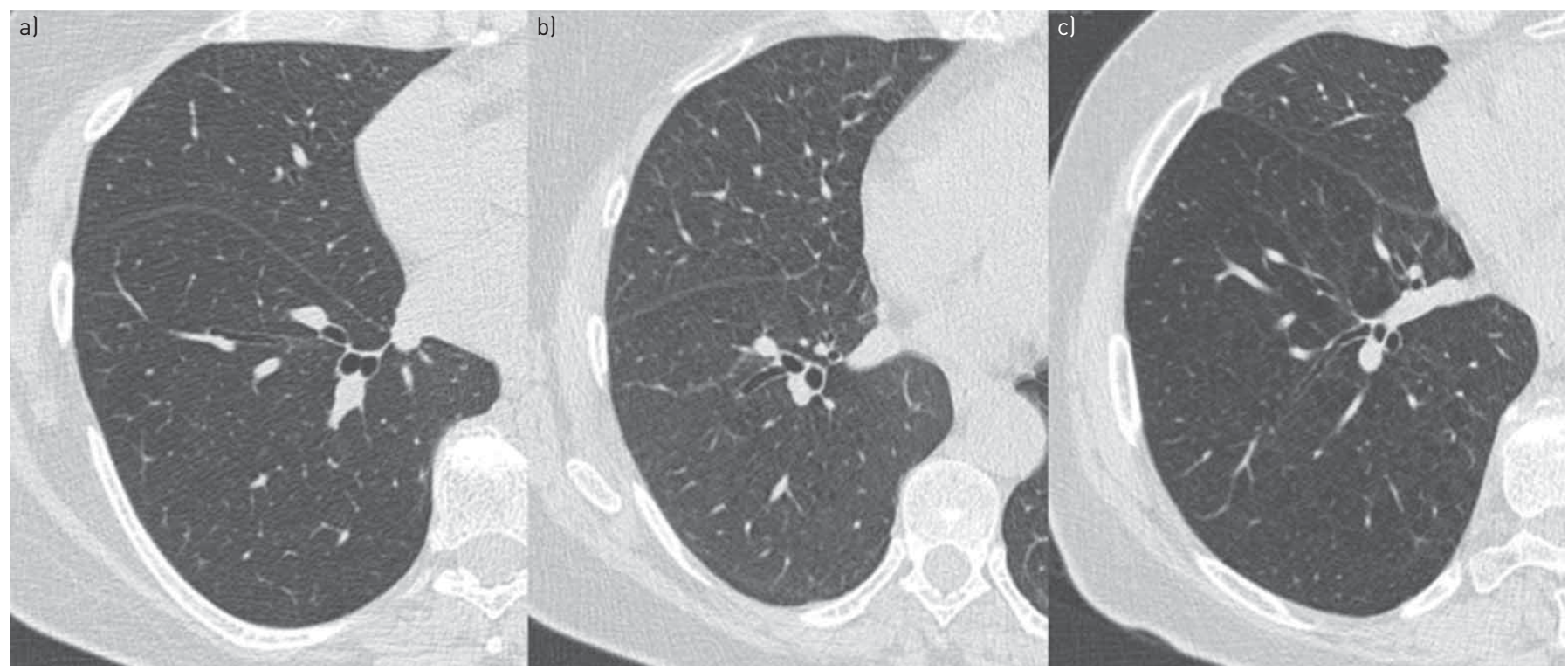

FIGURE 1 Axial inspiratory computed tomography (CT) scans at the level of the right inferior pulmonary vein, from three 54-year-old females, showing typical patterns. a) A healthy never-smoker. b) A smoker (note the subtle uniform diffuse increased opacity, which makes vessels more visible, and mild interlobular septal thickening, especially in middle lobe). c) A chronic obstructive pulmonary disease patient (note the diffusely distributed centrilobular emphysema, more in central part of the scans, with radiolucency and an irregular vascular pattern). Reproduced from [5] with permission from the publisher.

quantitatively providing pulmonary blood volume (PBV), pulmonary blood flow (PBF) and mean transit time. The normal lungs showed a significantly higher PBF and mean transit time $\left(154 \mathrm{~mL} \cdot 100 \mathrm{~mL} \cdot \mathrm{min}^{-1}\right.$ and $11 \mathrm{~s}$, respectively) compared with the abnormal lungs $\left(95 \mathrm{~mL} \cdot 100 \mathrm{~mL}^{-1} \cdot \mathrm{min}^{-1}\right.$ and $6.6 \mathrm{~s}$, respectively). Further insights into the perfusion distribution between gravitational dependent and isogravitational lung regions were provided. This study demonstrated, for the first time, a reasonable CT approach for assessment of whole lung perfusion information. Thus, dynamic volume perfusion CT may offer an opportunity to evaluate the morphological and functional features of the whole lung in a single examination.

Perfusion CT was applied to $63 \mathrm{COPD}$ patients to assess regional functional information in conjunction with morphological information [7]. 15 patients showed no CT morphological evidence of emphysema; however, perfusion defects were already noted. The size and distribution of perfusion defects in the other 48 COPD patients were consistent with the emphysema on the non-enhanced images. The rate of positive perfusion defects on the CT perfusion images was higher than that of emphysematous and bronchial abnormalities on the non-enhanced images (63 out of 63 versus 48 out of 63). The density measurements in the normal lung did not correlate with the pulmonary function test measurements, while density measurements in the area with perfusion defects correlated positively with forced expiratory volume in $1 \mathrm{~s}$ (FEV1) \% predicted $(\mathrm{r}=0.47 ; \mathrm{p}<0.001)$. This study demonstrated the excellent combination of a high spatial resolution CT dataset and a high spatial resolution perfusion dataset, so far unattained using other imaging techniques. This study for showed, the first time, a direct comparison between morphology and function in one setting. By contrast, magnetic resonance perfusion techniques have to be matched with CT images providing two different levels of resolution. Overall, perfusion imaging was more sensitive in detecting emphysema which is inconspicuous on CT images.

Despite the superior spatial resolution and image contrast for imaging of the lung parenchyma CT is associated with a relevant radiation dose. Although the CT perfusion results discussed earlier are promising, more and more investigations being performed using magnetic resonance perfusion for functional assessment of the lung parenchyma. BRYANT et al. [8] investigated the perfusion pattern in severe COPD patients (GOLD stage III/IV) and correlated these with CT based morphological lung parenchyma characteristics. A centrilobular type of emphysema was found to be characterised by a heterogeneous reduction in perfusion with focal defects (fig. 2). In panlobular emphysema the perfusion is more severely impaired leading to a loss of perfusion, either heterogeneously or homogeneously. Overall, magnetic resonance pulmonary perfusion patterns were found to correlate with the CT phenotype at a segmental level in patients with severe emphysema. Thus, the type of emphysema can be deduced using radiation free magnetic resonance perfusion assessment.

In the clinical scenario, assessment of perfusion is important in the context of endobronchial valve treatment of COPD. It is known that patients with heterogeneous emphysema are not optimal candidates 


\section{a)}
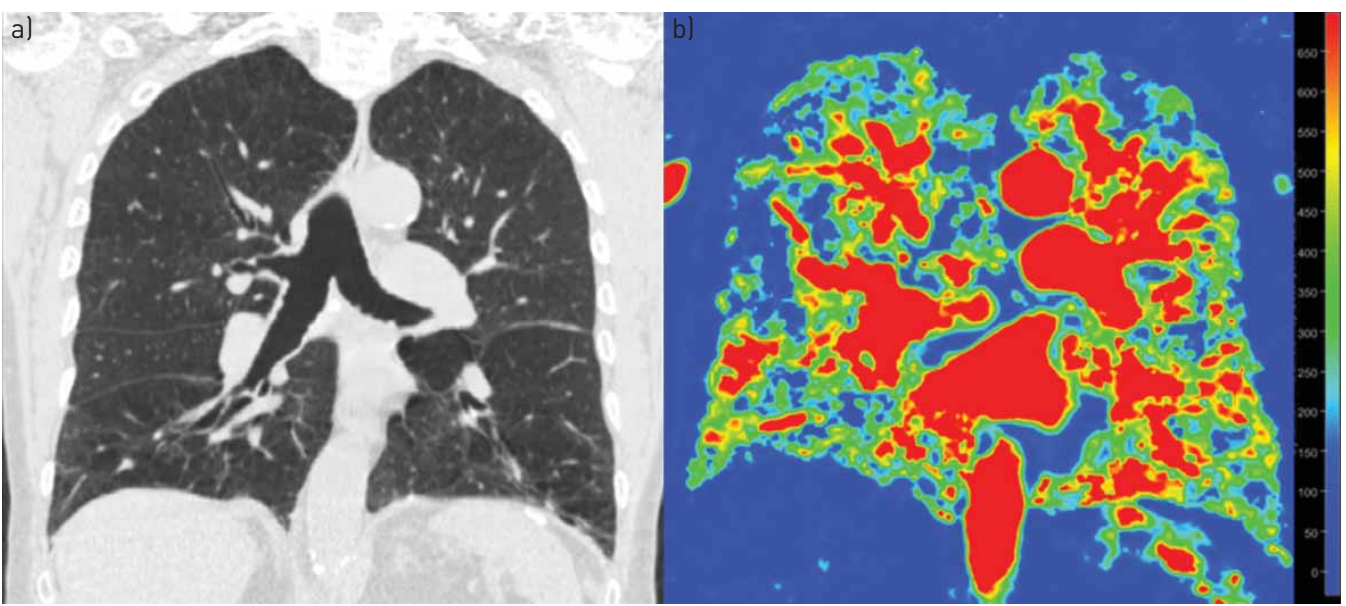

FIGURE 2 a) For segments in the middle and lower lobes centrilobular emphysema was found to be the predominant type on computed tomography. b) The corresponding colour-coded map of maximum peak enhancement of magnetic resonance perfusion showed heterogeneous perfusion with defects as the predominant pattern. Reproduced from [8] with permission from the publisher.

for endobronchial valve treatment. However, if target lobes with a low baseline perfusion were chosen a significant improvement in exercise tolerance was observed. 193 patients with heterogeneous emphysema were enrolled in a study assessing the impact of local perfusion (assessed by scintigraphy) on endobronchial valve outcome [9]. 88 patients showed low baseline target lobe perfusion and 81 patients had a high baseline target lobe perfusion. Patients with low baseline perfusion had a significantly greater improvement in 6-min walking distance than patients with a high baseline perfusion $(30.24 \mathrm{~m} ; \mathrm{p}=0.03)$.

\section{Imaging in oncology}

In 2011, a consensus paper from the leading lung cancer, thoracic and respiratory societies was published with major changes to the classification of adenocarcinomas [10]. This new classification strategy is based on a multidisciplinary approach to diagnosis of lung adenocarcinoma that incorporates clinical, molecular, radiological and surgical issues, but it is primarily based on histology. The terms bronchioloalveolar carcinoma (BAC) and mixed subtype adenocarcinoma are no longer used. Adenocarcinoma in situ (AIS) and minimally invasive adenocarcinoma (MIA) are the terms used for small solitary adenocarcinomas with either pure lepidic growth (AIS) or predominant lepidic growth with $\leqslant 5 \mathrm{~mm}$ invasion (MIA) to define patients who, if they undergo complete resection, will have $100 \%$ or nearly $100 \%$ disease-specific survival, respectively. AIS and MIA are usually nonmucinous, but on rare occasions may be mucinous. Invasive adenocarcinomas are classified by their predominant pattern after using comprehensive histological subtyping with lepidic (formerly most mixed subtype tumours with nonmucinous BAC), acinar, papillary and solid patterns; micropapillary has been added as a new histological subtype. Variants include invasive mucinous adenocarcinoma (formerly known as mucinous BAC), colloid, fetal, and enteric adenocarcinoma. This classification provides guidance on small biopsies and cytology specimens, as $\sim 70 \%$ of lung cancers are diagnosed in such samples [10].

This new guideline is highly important as it redefines survival rates of patients. However, it challenges radiology as it is difficult to discriminate between the entities noninvasively. The radiological differentiation between invasive pulmonary adenocarcinoma and pre-invasive lesions appearing as ground-glass nodules (GGN) was investigated in 253 patients by LeE et al. [11]. 64 pure GGNs and 208 part-solid nodules were found in this population. In pure GGNs, pre-invasive lesions were significantly smaller and more frequently nonlobulated than invasive lesions. Lesion size was the single significant differentiator of pre-invasive lesions from invasive lesions, with $10 \mathrm{~mm}$ being the optimal cut-off size for pre-invasive lesions. In part-solid GGNs, there were significant differences in lesion size, solid portion size, solid proportion, margin, border and pleural retraction between invasive and pre-invasive lesions. To differentiate between noninvasive and invasive lesions a lesion size of $\leqslant 14 \mathrm{~mm}$ (sensitivity $67 \%$ and specificity $74 \%$ ) and a solid portion of $\leqslant 29 \%$ (sensitivity $69 \%$ and specificity $80 \%$ ) were found to be suitable cut-off values.

In daily clinical practice, the lesion size of GGNs is usually smaller than $10 \mathrm{~mm}$. To date, the optimal follow-up regimen is unknown. In a follow-up study of 122 GGNs for more than 2 years a $9.8 \%$ frequency of growth was found on a per nodule basis [12]. The median volume doubling time was 769 days. Nodule 
growth was significantly associated with initial size and new development of an internal solid portion. Most of the growing nodules (11 out of 12 ) were confirmed as primary lung cancer. In conclusion, $90 \%$ of the pure GGNs in the lung did not grow during long-term follow-up in subjects with no history of malignancy and most growing nodules had an indolent clinical course.

These data demonstrate the difficulties in making recommendations about the optimal follow-up period. This is further complicated by a study by KANEDA et al. [13] who followed 32 GGNs (26 pure GGNs) for 21 months (median time interval) before surgery. 47\% of the GGNs showed a size reduction on follow-up chest CT. $58 \%$ of pure GGNs remained pure GGNs and the remainder developed a solid component. The authors concluded that a size reduction suggested progression to an invasive adenocarcinoma. When a mild collapse of the GGN is observed, a careful follow-up is necessary to identify a solid component. Tumour size decreases may represent the optimal timing of pulmonary resection for curative treatment.

Given the aforementioned studies and further studies not discussed in this review, the management of non-solid and part solid lesions is difficult. Currently, there are two different published recommendations, one from the Fleischner Society [14] and one from the American College of Chest Physicians (ACCP) [15]. The main differences between the two recommendations are the lesion size cut-off values for follow-up imaging and pathology.

The Fleischner Society recommends no follow-up imaging for a solitary pure GGN $\leqslant 5 \mathrm{~mm}$ [14]. For lesions $>5 \mathrm{~mm}$, an initial follow-up CT at 3 months is recommended to confirm persistence. This is followed by an annual CT for a minimum of 3 years. In part solid nodules an initial follow-up CT at 3 months is recommended to confirm persistence. If the nodule is persistent and the solid component is $<5 \mathrm{~mm}$, then an annual CT for a minimum of 3 years is recommended. If the nodule is persistent and the solid component is $\geqslant 5 \mathrm{~mm}$ a biopsy or surgical resection should be carried out.

The ACCP recommendations for a solitary pure GGN $\leqslant 5 \mathrm{~mm}$ are no follow-up imaging [15]. For lesions $>5 \mathrm{~mm}$ an annual CT for a minimum of 3 years should be performed. For lesions $>10 \mathrm{~mm}$ an initial follow-up CT at 3 months is recommended to confirm persistence; if the lesion is persistent biopsy/ resection is recommended. In part solid nodules $\leqslant 8 \mathrm{~mm}$ CT surveillance is recommended at 3,12 and 24 months, followed by annual surveillance for $1-3$ years. If the overall lesion is $>8 \mathrm{~mm}$ CT should be performed after 3 months to confirm persistence and subsequent biopsy/resection.
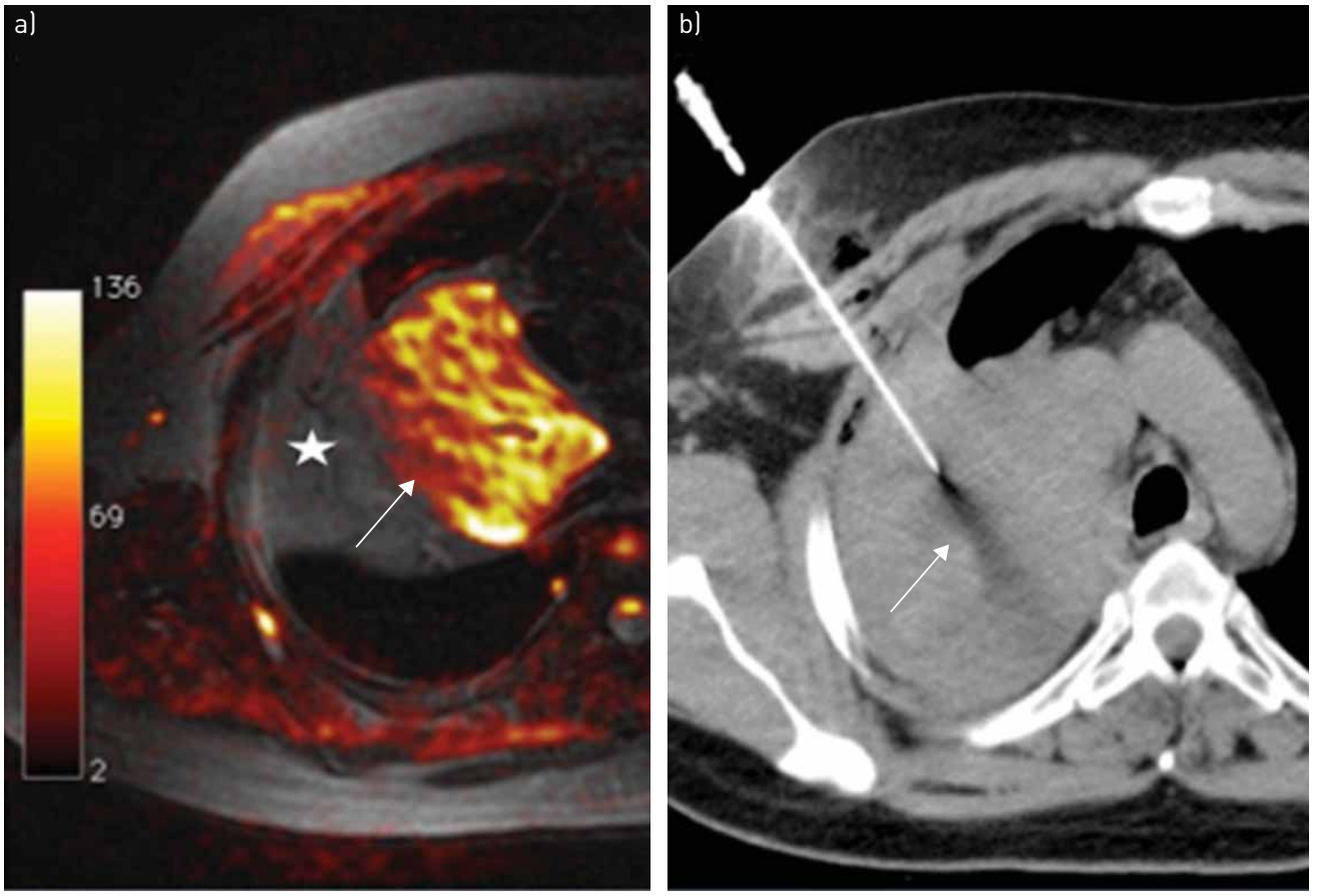

FIGURE 3 Images from a 62-year-old male patient presenting with a heterogeneous mass in the superior lobe of the right lung. a) Fusion of axial fat-saturated T1-weighted and diffusion-weighted sequences. Magnetic resonance imaging (MRI) demonstrated that only the anteromedial portion (arrow) of the mass showed high signal intensity on the diffusion-weighted MRI sequence, suggesting that the rest of the mass may be composed of atelectasis, consolidation or necrosis $\left({ }^{*}\right)$. b) Computed tomography guided biopsy was directed to this area, and histopathological examination yielded a diagnosis of lung adenocarcinoma. Reproduced from [16] with permission from the publisher. 
Given the complexity of these lesions, the current recommendations are more varied than in other areas. It cannot be overemphasised that these guidelines must be interpreted in light of an individual's clinical history and will need to be adapted with the gain of new scientific insights [14].

Large (central) lung carcinomas with subsequent areas of atelectasis are challenging as it is difficult to determine the border between the tumour and atelectasis. This is especially important in determining the right location for taking a biopsy. For differentiation between atelectasis and a tumour functional information are required, which can be gained using PET or MRI. In a study including 13 patients with lung lesions and adjacent atelectasis functional information were used to target the optimal area for a lung biopsy [16]. For MRI the diffusion-weighted technique was used and evaluated using the apparent diffusion coefficient. All biopsies yielded adequate material for histopathological diagnosis (fig. 3). The biopsy areas showed higher diffusion-weighted signal intensity and lower apparent diffusion coefficients, suggesting a high cellularity. In patients who underwent PET/CT, areas with higher 18-fluorodeoxyglucose concentrations corresponded to areas of higher diffusion-weighted signal intensity and lower apparent diffusion coefficients. In clinical practice the assessment of functional information is useful for lung biopsy planning to avoid false negative biopsies. The use of MRI or PET/CT is equally possible.

\section{Conclusion}

Several major topics have been addressed by pulmonary imaging the past 12 months. In particular, the importance of the interaction between anatomy (lung fissures) and function (perfusion) has to be highlighted for guidance of endobronchial treatment in emphysema. In addition, the recently changed classification of non-solid and part-solid pulmonary lesions and the implications for management have been demonstrated.

\section{References}

1 Sciurba FC, Ernst A, Herth FJ, et al. A randomized study of endobronchial valves for advanced emphysema. N Engl J Med 2010; 363: 1233-1244.

2 Koenigkam-Santos $\mathrm{M}$, de Paula WD, Owsijewitsch $\mathrm{M}$, et al. Incomplete pulmonary fissures evaluated by volumetric thin-section CT: semi-quantitative evaluation for small fissure gaps identification, description of prevalence and severity of fissural defects. Eur J Radiol 2013; 82: 2365-2370.

$3 \mathrm{Pu} \mathrm{J}$, Wang Z, Gu S, et al. Pulmonary fissure integrity and collateral ventilation in COPD patients. PLoS One 2014; 9: e96631.

4 Smith BM, Hoffman EA, Rabinowitz D, et al. Comparison of spatially matched airways reveals thinner airway walls in COPD. The Multi-Ethnic Study of Atherosclerosis (MESA) COPD Study and the Subpopulations and Intermediate Outcomes in COPD Study (SPIROMICS). Thorax 2014; 69: 987-996.

5 Karimi R, Tornling G, Forsslund H, et al. Lung density on high resolution computer tomography (HRCT) reflects degree of inflammation in smokers. Respir Res 2014; 15: 23.

6 Sun $\mathrm{H}, \mathrm{Gao}$ F, Li N, et al. An evaluation of the feasibility of assessment of volume perfusion for the whole lung by 128-slice spiral CT. Acta Radiol 2013; 54: 921-927.

7 Guan Y, Xia Y, Fan L, et al. Quantitative assessment of pulmonary perfusion using dynamic contrast-enhanced CT in patients with chronic obstructive pulmonary disease: correlations with pulmonary function test and CT volumetric parameters. Acta Radiol 2015; 56: 573-580.

8 Bryant M, Ley S, Eberhardt R, et al. Assessment of the relationship between morphological emphysema phenotype and corresponding pulmonary perfusion pattern on a segmental level. Eur Radiol 2015; 25: 72-80.

9 Argula RG, Strange C, Ramakrishnan V, et al. Baseline regional perfusion impacts exercise response to endobronchial valve therapy in advanced pulmonary emphysema. Chest 2013; 144: 1578-1586.

10 Travis WD, Brambilla E, Noguchi M, et al. International Association for the Study of Lung Cancer/American Thoracic Society/European Respiratory Society international multidisciplinary classification of lung adenocarcinoma. J Thorac Oncol 2011; 6: 244-285.

11 Lee SM, Park CM, Goo JM, et al. Invasive pulmonary adenocarcinomas versus preinvasive lesions appearing as ground-glass nodules: differentiation by using CT features. Radiology 2013; 268: 265-273.

12 Chang B, Hwang JH, Choi YH, et al. Natural history of pure ground-glass opacity lung nodules detected by low-dose CT scan. Chest 2013; 143: 172-178.

13 Kaneda H, Nakano T, Taniguchi Y, et al. A decrease in the size of ground glass nodules may indicate the optimal timing for curative surgery. Lung Cancer 2014; 85: 213-217.

14 Naidich DP, Bankier AA, MacMahon H, et al. Recommendations for the management of subsolid pulmonary nodules detected at CT: a statement from the Fleischner Society. Radiology 2013; 266: 304-317.

15 Gould MK, Donington J, Lynch WR, et al. Evaluation of individuals with pulmonary nodules: when is it lung cancer? Diagnosis and management of lung cancer, 3rd ed: American College of Chest Physicians evidence-based clinical practice guidelines. Chest 2013; 143: e93S-e120S.

16 Guimaraes MD, Marchiori E, Odisio BC, et al. Functional imaging with diffusion-weighted MRI for lung biopsy planning: initial experience. World J Surg Oncol 2014; 12: 203. 